\title{
Fine structure in sunspots
}

\section{Penumbral grains in speckle reconstructed images}

\author{
M. Sobotka ${ }^{1}$ and P. Sütterlin ${ }^{2}$ \\ 1 Astronomical Institute, Academy of Sciences of the Czech Republic, 25165 Ondřejov, Czech Republic \\ 2 Sterrenkundig Instituut Utrecht, Postbus 80000 , 3508 TA Utrecht, The Netherlands \\ e-mail: P.Suetterlin@astro.uu.nl
}

Received 4 July 2001 / Accepted 15 October 2001

\begin{abstract}
The properties of penumbral grains (PGs) in a large regular sunspot are studied from a 70 min sequence of $G$-band images acquired on 20 September 1999 at the Dutch Open Telescope, La Palma. The frames were processed using the speckle masking algorithm, resulting in an almost diffraction-limited time series $(30 \mathrm{~s}$ cadence), basically free of atmospheric distortions. Applying feature tracking to a movie of 140 frames yields proper motions, intensities, and lifetimes for a set of 1058 PGs with lifetimes longer than 10 min. About $54 \%$ of the PGs move toward the umbra and $46 \%$ toward the photosphere. The inward-moving PGs are located mostly in the inner penumbra (up to 0.6 of the distance from the umbra to the photosphere). Their average lifetime and median speed are $50 \mathrm{~min}$ and $0.52 \mathrm{~km} \mathrm{~s}^{-1}$. Most of the outward-moving PGs are observed in the outer penumbra and their average lifetime and median speed are $31 \mathrm{~min}$ and $0.75 \mathrm{~km} \mathrm{~s}^{-1}$. These measurements confirm the previous results published by Sobotka et al. (1999a).
\end{abstract}

Key words. Sun: sunspots

\section{Introduction}

Bright filaments in sunspot penumbrae are, in fact, chains of elongated bright features called penumbral grains (PGs, Muller 1973a,b). PGs are dynamical objects. Some discrepancies concerning their lifetimes and horizontal motions can be found in the literature. For example, Muller (1973a) and Tönjes \& Wöhl (1982) reported lifetimes of 1-3 hours and motions mostly toward the umbra. Wang \& Zirin (1992) and Denker (1998), using local correlation tracking, found inward horizontal flows in the inner penumbra but outward motions (toward the photosphere) in the outer parts. Sobotka et al. (1999a, hereafter Paper III) applying a feature tracking algorithm, detected both inward (INW) and outward (OUT) moving PGs with considerably shorter mean lifetimes: 39 (INW) and 25 (OUT) minutes. They found a dividing line in the penumbra, approximately 0.7 of the distance from the umbra to the photosphere, such that most PGs outside this line moved to the photosphere, and those inside moved towards the umbra.

Send offprint requests to: M. Sobotka, e-mail: msobotka@asu.cas.cz
In this work we apply a similar technique as in Paper III to a series of speckle reconstructed images of another sunspot, taken with a different telescope and in a different wavelength band, in order to check the previous results.

\section{Observations and data reduction}

The observations were acquired on September 20, 1999 with the Dutch Open Telescope (DOT) at the Observatorio del Roque de Los Muchachos on La Palma (Hammerschlag \& Bettonvil 1998). This dome-less open telescope of $44 \mathrm{~cm}$ aperture yields a scale of $103 . .3 / \mathrm{mm}$ in the focal plane. The image is enlarged by a magnification system to $9.18 / \mathrm{mm}$ in the image plane, where a video camera $(768 \times 572$ pixel $)$ records the images at a frame rate of approximately $7-8$ frames/s and a spatial sampling of 0 '.081/pixel (FOV $62.5 \times 46 \operatorname{arcsec}^{2}$ ). The exposure time was $1 \mathrm{~ms}$ using a bandpass of $1 \mathrm{~nm}$ centered at $430.5 \mathrm{~nm}$ ( $G$-band). The video signal was digitized by an 8-bit frame grabber device in a PC. Data were taken in bursts of 100 images, and were then written to hard disk. A new burst was started every $30 \mathrm{~s}$. After the observation, the recorded data were transferred to DAT tape. 


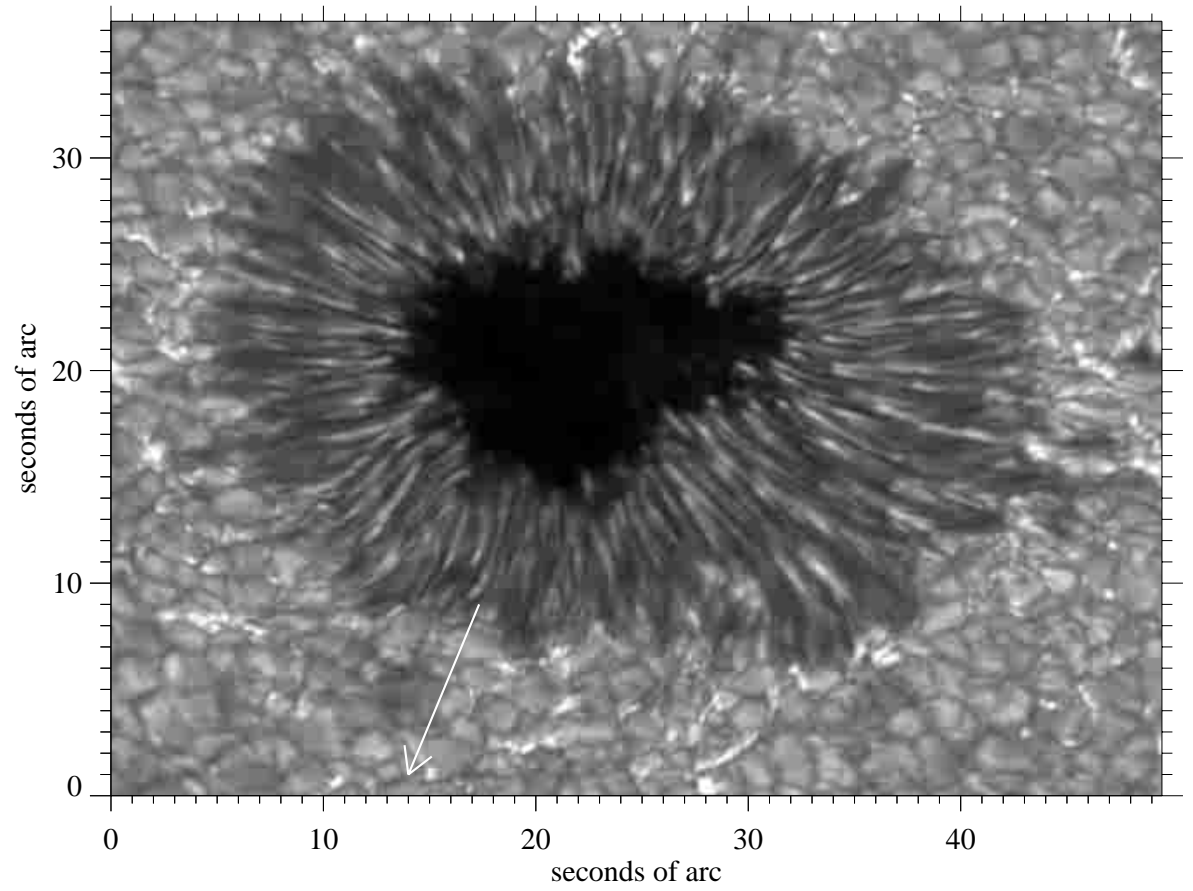

Fig. 1. A speckle reconstructed image of sunspot NOAA 8704. The arrow marks the direction towards disk center.
A large regular spot (NOAA 8704) at the heliocentric position S22 E32 $(\mu=0.76)$ was observed from 10:34 to $13: 35$ UT. The first part of the series, from 10:34 to 11:44 UT, was corrected for the instrumental profile of the telescope and for the influences of atmospheric seeing using the speckle masking algorithm (Weigelt \& Wirnitzer 1983; von der Lühe 1993; de Boer 1993). The true aperture, including obstructions in the optical path of the telescope, has been included in the speckle reconstruction code. Due to its simple optical layout, DOT is an extremely low-scattering telescope. The effect of scattered light can be neglected, as seen from observed umbral minimum intensities (about 0.08 of the mean intensity of the quiet photosphere). The seeing conditions during that period were excellent, as shown by the average Fried parameter (measured at the observation wavelength) of $r_{0}=11 \mathrm{~cm}$ with extremal values of $7.2 \mathrm{~cm}$ and $14.6 \mathrm{~cm}$. Due to storage limitations, the second part of the series was not taken in bursts but in frame-selection mode, so that no speckle reconstruction was possible. All specklereconstructed images have been correlated and clipped to the common field of view of $49 \times 36 \operatorname{arcsec}^{2}$. The DOT has a parallactic mount, therefore, no image de-rotation was necessary.

Due to the limited sample size of the bursts, the quality of the reconstruction is still somewhat dependent on the atmospheric conditions. Therefore, in an additional step, the power spectra of all images of the series have been enhanced so that the azimuthally averaged power distribution resembles that of the best image in the series. To the resulting time series we applied a subsonic $k-\omega$ filter with a cutoff velocity of $7 \mathrm{~km} \mathrm{~s}^{-1}$ to remove the effects of the 5-min oscillation. This process resulted in a series of 140 reconstructed frames with a constant time lag equal to $30 \mathrm{~s}$. A typical frame is shown in Fig. 1.
All frames were then resampled to compensate for the geometrical distortion due to the position of the spot at $\mu=0.76$. To remove a residual jitter (stemming from the division of the images into isoplanar subfields), the series was smoothed in time by means of a boxcar spanning over 3 frames.

To isolate PGs from other structures, we masked out photospheric granules, eliminated umbral dots by setting the minimum intensity of bright features to 0.5 of the mean intensity of the quiet photosphere, and applied a segmentation algorithm based on a search for regions with convex intensity profiles. A feature tracking algorithm, described by Sobotka et al. (1997), was applied to the series of segmented frames to follow each $P G$ in time. To be called a PG, each feature had to have a lifetime of at least $3 \mathrm{~min}$, a minimum mean area over its lifetime of at least 16 pixels, and a time-averaged velocity smaller than $2 \mathrm{~km} \mathrm{~s}^{-1}$.

This procedure yielded a set of 2900 PGs. Since the duration of the series $(70 \mathrm{~min})$ was shorter than lifetimes of many long-lived PGs (114 PGs were observed during the full period), we were limited only to a statistical estimate of the mean lifetime based on an average birth rate of PGs (see Sobotka et al. 1999b, for details).

Time-averaged horizontal velocities were calculated using linear least-squares fits to the positions of PGs. To increase the accuracy of velocity determination, we chose for analysis 1489 PGs with lifetimes longer than $10 \mathrm{~min}$. In this new set, the velocity is measured with a standard deviation of about $0.05 \mathrm{~km} \mathrm{~s}^{-1}$. In the last step of the data analysis we checked visually the trajectories of these 1489 PGs to eliminate spurious objects and mistakes in feature tracking (cf. Paper III). After this visual consistency check we obtained a final sample of 1058 PGs. 


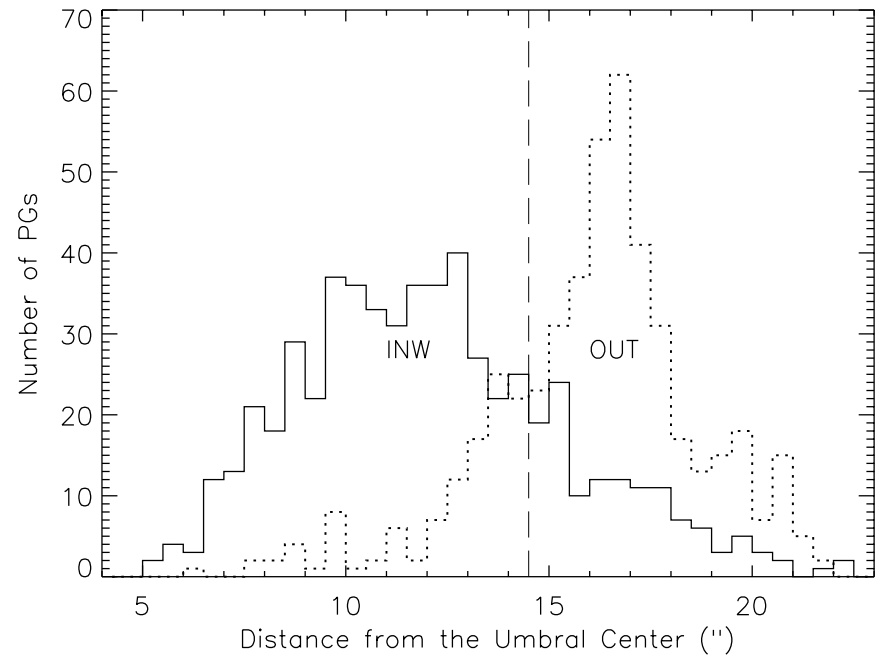

Fig. 2. Histogram of time-averaged distances of the INW (solid line) and OUT (dotted line) PGs from the center of the umbra. The position of the dividing line is marked by vertical dashes.

\section{Results}

Like in the case described in Paper III, both INW and OUT PGs were observed. Of the 2900 detected PGs, 1477 moved inward toward the umbra, 1407 outward toward the photosphere, and 16 were stationary or had a tangential motion. Of the 1058 PGs that passed the visual consistency check, 575 (54\%) moved inward and 483 (46\%) outward.

\subsection{Estimated mean lifetimes}

Considering all 2900 detected PGs (minimum lifetime set to $3 \mathrm{~min}$ ), the estimated mean lifetimes are $25.2 \mathrm{~min}$ (INW) and $18.4 \mathrm{~min}$ (OUT). These values are very similar to the estimates (INW: 27, OUT: $19 \mathrm{~min}$ ) obtained in Paper III for all the observed PGs.

Based on the final sample of 1058 PGs (minimum lifetime set to $10 \mathrm{~min}$ ), the estimated mean lifetimes are 50.3 and $30.8 \mathrm{~min}$ for INW and OUT PGs, respectively. These values are by about $25 \%$ higher than those presented in Paper III for the final sample (INW: 39, OUT: $25 \mathrm{~min}$ ). The lifetimes of both INW and OUT PGs depend on the position in the penumbra: The average lifetime near to the penumbra-umbra boundary is $55 \mathrm{~min}$, it reaches the maximum, $70 \mathrm{~min}$, at about $1 / 4$ of the width of the penumbra and then it decreases gradually to $30 \mathrm{~min}$ at the outer penumbral border. This trend is consistent with the result shown in Paper III.

\subsection{Spatial distribution and trajectories}

The final sample of 1058 PGs was analyzed to obtain the spatial distribution of positions and trajectories. The histograms of time-averaged distances of the INW and OUT PGs from the center of the umbra are plotted in Fig. 2.

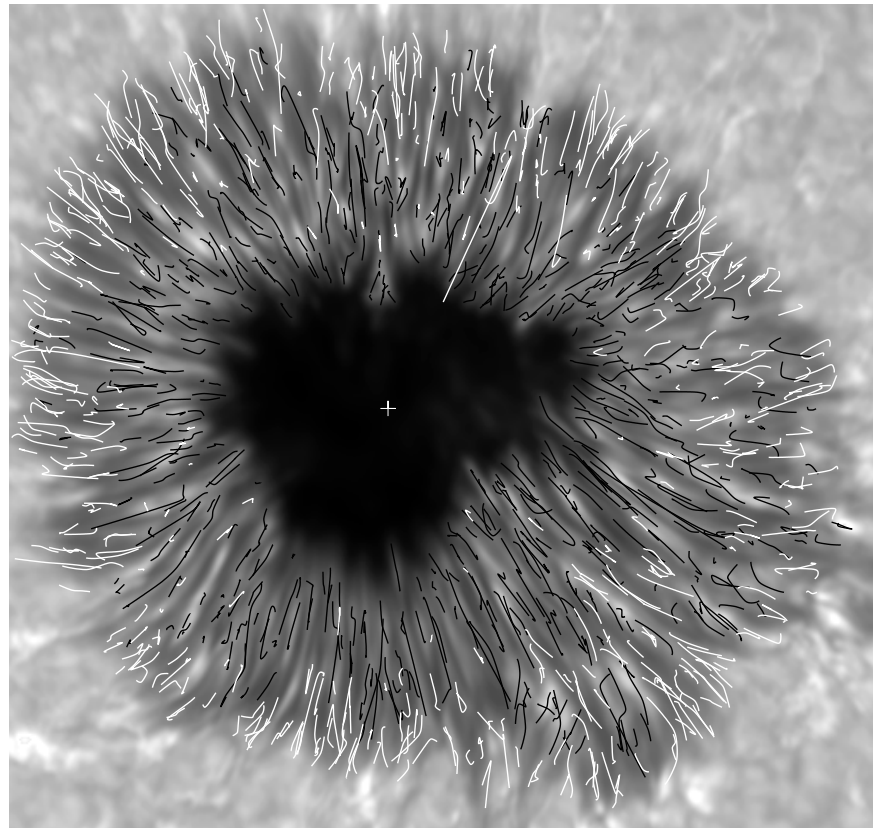

Fig. 3. Trajectories of INW (black) and OUT (white) PGs. The underlying image is an average of all frames in the series. The center of the umbra is marked by a white cross. The image size is $29.8 \times 28.4 \mathrm{Mm}$.

The INW PGs dominate in the distance range $5^{\prime \prime}-14^{\prime \prime} 5$, i.e. in the inner penumbra, while the OUT PGs dominate in the range $14^{\prime \prime} .5-22^{\prime \prime}$ (outer penumbra). The distance $14^{\prime \prime} .5$ from the umbral center can be considered as a dividing line between the regions of prevailing INW or OUT PGs. In units of a relative distance in the penumbra, measured from an average position of the penumbraumbra border (position 0) to an average position of the penumbra-photosphere boundary (position 1), the dividing line is located at about 0.6 .

The trajectories of PGs were determined from the positions tracked in time and smoothed by cubic splines. In Fig. 3 we show trajectories of the INW and OUT PGs as black and white lines, respectively. The average length of trajectories is 1". 3 for both INW and OUT PGs (similar values are given in Paper III). Only $16 \%$ INW and $19 \%$ OUT PGs traveled longer than $2^{\prime \prime}$. The maximum observed length of trajectory is $6^{\prime \prime}$ ( 2 INW and 1 OUT PGs) in a penumbra with an average width of $12^{\prime \prime}$, so none of the PGs crossed the penumbra completely. Figure 3 seems to indicate a much higher amount of long trajectories. However, most of them are formed by overlapping paths of PGs that appeared in the same area, but at different times.

\subsection{Horizontal velocities}

The time-averaged horizontal velocities of the 575 INW PGs, included in the final sample, show an asymmetric histogram with a maximum around $0.4 \mathrm{~km} \mathrm{~s}^{-1}$, a mean of $0.60 \mathrm{~km} \mathrm{~s}^{-1}$, and a median of $0.52 \mathrm{~km} \mathrm{~s}^{-1}$. Velocities of 


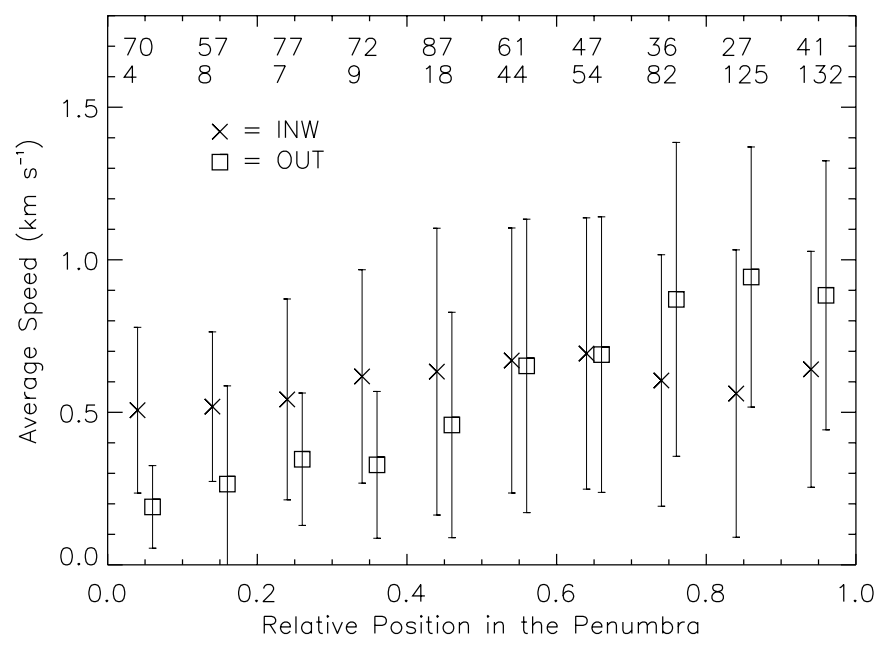

Fig. 4. Average speed of PGs as a function of their relative positions in the penumbra. INW PGs are represented by $\times$, OUT PGs by $\square$. Position 0.0 is at umbra, 1.0 at photosphere. The bars indicate a scatter of $\pm 1 \sigma$. Numbers of INW (OUT) $\mathrm{PGs}$ in each bin are displayed in the upper (lower) row.

the 483 OUT PGs have a broader histogram than that of INW, with a maximum around $0.5 \mathrm{~km} \mathrm{~s}^{-1}$, a mean of $0.80 \mathrm{~km} \mathrm{~s}^{-1}$, and a median of $0.75 \mathrm{~km} \mathrm{~s}^{-1}$. These speeds are only slightly higher than those presented in Paper III.

To study the dependence of time-averaged velocities on position, we divided the relative distances in the penumbra into 10 bins. Velocities of all PGs whose time-averaged positions fell into a given bin were averaged. The results are plotted in Fig. 4 together with $1 \sigma$ error bars (which characterize the scatter of individual values) and with numbers of PGs in each bin. For the INW PGs speeds increase from $0.5 \mathrm{~km} \mathrm{~s}^{-1}$ at the penumbra-umbra boundary to a maximum of $0.7 \mathrm{~km} \mathrm{~s}^{-1}$ at the relative distance $0.6-0.7$ (just outside the dividing line) and then drop to $0.6 \mathrm{~km} \mathrm{~s}^{-1}$ in the outer penumbra. Speeds of the OUT PGs increase from a minimum value of $0.2 \mathrm{~km} \mathrm{~s}^{-1}$ at the penumbraumbra border to a maximum of $0.9 \mathrm{~km} \mathrm{~s}^{-1}$ near the outer penumbral boundary. These velocity distributions are very similar to those obtained in Paper III.

We calculated instantaneous velocities of each $\mathrm{PG}$ as derivatives of positions smoothed by cubic splines. Individual velocity curves, showing acceleration/deceleration, were divided into four types and corresponding numbers of PGs were counted (cf. Table 1). These numbers are in a good agreement with those published in Paper III. Most of INW PGs (60.5\%, types 2 and 4) decelerate at least in the initial phase of their life, which is partially consistent with the prediction given in the model by Schlichenmaier et al. (1998).

\section{Discussion and conclusions}

In this work we extend the study of lifetimes and horizontal motions of PGs, described in Paper III, using
Table 1. Numbers of PGs by velocity type.

\begin{tabular}{cccc}
\hline & & INW & OUT \\
\hline 1 Acceleration & $144(25 \%)$ & $143(30 \%)$ \\
2 Deceleration & $215(37.5 \%)$ & $141(29 \%)$ \\
3 & $83(14.5 \%)$ & $110(23 \%)$ \\
$\begin{array}{c}\text { Acceleration, } \\
\text { then deceleration }\end{array}$ & $133(23 \%)$ & $89(18 \%)$ \\
\hline $\begin{array}{c}\text { Deceleration, } \\
\text { then acceleration }\end{array}$ & & \\
\hline
\end{tabular}

observations and image processing that differ in the following points:

- The spot NOAA 8704 was large and nearly circular. The sunspot studied in Paper III was medium-size and had four umbral cores. In Paper III, we mentioned that the character of the motions of PGs may vary with the size and evolutionary stage of a spot. Therefore, it is important to compare the results obtained from observations of different sunspots.

- The wavelength is different - G-band at $\lambda 430.5 \mathrm{~nm}$ instead of $\lambda 468 \mathrm{~nm}$.

- The images are speckle-reconstructed, so that not only the instrumental profile of the telescope but also the influence of atmospheric blurring is corrected. This results in a very high and stable image quality during the whole time series.

- The segmentation algorithm, based on a search for local intensity maxima, differs from that used in Paper III, which applied a variable intensity threshold computed as a function of image sharpness.

- The final sample of PGs is more than twice larger than that analyzed in Paper III.

In spite of all the differences mentioned above, the results of our present analysis are consistent with those shown in Paper III. The main differences are in the estimated mean lifetimes that are by about $25 \%$ longer and in the larger fraction of OUT PGs (46\% present work, 27\% Paper III). The relative position of the dividing line between the regions of prevailing INW or OUT PGs is 0.6 instead of 0.7 . All the other results do not show any important difference. We can conclude that, using a new and independent observation, we confirm the previous results published in Paper III.

Schlichenmaier et al. (1998) suggested that PGs are intersections of hot, rising fluxtubes with the photosphere. They move toward the umbra, continuously decreasing their speed. The predictions of this model are only in partial agreement with our observations. Further numerical simulations are needed to account for interactions of several fluxtubes and to explain both the inward and outward motions of PGs. 
Acknowledgements. The work of M. Sobotka was accomplished under Grant A 3003903 by the Grant Agency of the Academy of Sciences of the Czech Republic (ASCR) and under the Key Project K 2043105 of the ASCR. P. Sütterlin is funded by the European Solar Magnetometry Network (ESMN) under EC-TMR contract ERBFMRXCT98019. The DOT project is funded by Utrecht University, The Netherlands Graduate School for Astronomy NOVA and The Netherlands Organization for Scientific Research NWO.

\section{References}

de Boer, C. R. 1993, Ph.D. Thesis, Universität Göttingen Denker, C. 1998, Sol. Phys., 180, 81
Hammerschlag, R. H., \& Bettonvil, F. C. M. 1998, New Astron. Rev., 42, 485

Muller, R. 1973a, Sol. Phys., 29, 55

Muller, R. 1973b, Sol. Phys., 32, 409

Schlichenmaier, R., Jahn, K., \& Schmidt, H. U. 1998, A\&A, 337,897

Sobotka, M., Brandt, P. N., \& Simon, G. W. 1997, A\&A, 328, 682

Sobotka, M., Brandt, P. N., \& Simon, G. W. 1999a, A\&A, 348, 621

Sobotka, M., Vázquez, M., Bonet, J. A., Hanslmeier, A., \& Hirzberger, J. 1999b, ApJ, 511, 436

Tönjes, K., \& Wöhl, H. 1982, Sol. Phys., 75, 63

von der Lühe, O. 1993, A\&A, 268, 374

Wang, H., \& Zirin, H. 1992, Sol. Phys., 140, 41

Weigelt, G., \& Wirnitzer, B. 1983, Opt. Lett., 8, 389 